\section{Osteoarthrose - nur symptomatische oder auch kausale Therapie?}

\author{
T. Pap ${ }^{1}$ \\ 1 Institut für Experimentelle Muskuloskelettale Medizin (IEMM), \\ Universitätsklinikum Münster
}

Die Osteoarthrose, im englischen Sprachraum wegen ihrer entzündlichen Komponente auch Osteoarthritis genannt, ist die häufigste Gelenkerkrankung. Sie betrifft mehr als die Hälfte aller 65-jährigen und stellt in unserer einerseits alternden, anderseits aber auf möglichst lange Mobilität des Einzelnen bedachten Gesellschaft eine zunehmende sozioökonomische Herausforderung dar. Dies gilt insbesondere, da es in den vergangenen zwanzig bis dreißig Jahren keine nennenswerten Fortschritte in der (medikamentösen) Therapie der Osteoarthrose gegeben hat. Eine Vielzahl von Studien vor allem zur Sekundär- und Tertiärprävention durch Verhaltensumstellung und Patientenaufklärung sowie zu abgestuften Therapierichtlinien können nicht darüber hinwegtäuschen, dass sich die pharmakologischen Möglichkeiten nach wie vor in der symptomatischen Beeinflussung von Schmerzen und Entzündungsschüben erschöpfen, und es Fortschritte vor allem in den operativen Therapieverfahren gegeben hat. Die Ursachen für fehlende Erfolge in der Entwicklung kausaler Behandlungsansätze sind vielschichtig, liegen vor allem aber in einem mangelnden Verständnis der Pathogenese der Osteoarthrose. Unsere mangelhaften Kenntnisse über die Mechanismen, die zur Entwicklung einer Osteoarthrose führen und diese gegen normale Alterung einerseits und gegen andere Gelenkerkrankungen andererseits abgrenzen, sind zu einem gewichtige Teil auch dafür verantwortlich, dass es gegenwärtig kaum verlässliche (Bio)Marker der Osteoarthrose gibt, die zur Diagnose, Verlaufskontrolle und zum Monitoring von Therapieerfolgen eingesetzt werden können. In der Folge fehlen nicht nur innovative Ansätze zur medikamentösen Krankheitsbeeinflussung, sondern auch die Instrumente, einen möglichen Erfolg krankheitsmodifizierender Therapien in klinischen Studien zu verfolgen.

Die Osteoarthrose hat eine primär degenerative Genese und führt zum progressiven Verlust des Gelenkknorpels. Dabei finden in erkrankten Gelenken komplexe Umbauvorgänge statt, die auch die Synovialmembran (Entzündungsschübe) und den Knochen (subchondrale Veränderungen, gelenknahen Osteophyten) einbeziehen. Jüngste Untersuchungen belegen, dass für die Entstehung der Osteoarthrose zwei Mechanismen von zentraler Bedeutung sind: Zum einen kommt es zu einer Reaktivierung embryonaler Reaktionsmuster in den Chondrozyten, die ursprünglich während der Knochenbildung durch enchondrale Ossifikation eine Rolle spielen. Dazu gehört vor allem die (hypertrophe bzw. De-)Differenzierung der Chondrozyten und die Re-Expression embryonal nachweisbarer Oberflächenmoleküle, die Ausgangspunkt für weitere Veränderungen sind. So konnten wir mit dem transmembranen Heparansulfatproteoglycan Syndcan-4 ein Molekül identifizieren, das offenbar eng mit der Reaktivierung embryonaler Differenzierungsmuster während der Osteoarthrose verbunden ist und deren Hemmung die Entstehung osteoarthrotischer Veränderungen verhindern kann. Mehrere andere Untersuchungen der vergangenen Jahre unterstützen die These von einer Verbindung zwischen Prozessen der enchondralen Ossifikation und der Osteoarthrose. So gibt es offenbar Gemeinsamkeiten in der (z.B. Hypoxie induzierten) Regulation knorpel- und knochenspezifischer Transkriptionsfaktoren. Außerdem zeigt sich, dass die bislang als Sonderfall der Arthrose angesehene Kalzifizierung des Gelenkknorpels untrennbar mit der Entstehung arthrotischer Veränderungen verbunden ist und dass - in Analogie zur enchondralen Ossifikation beim Embryo - Chondrozyten im Knorpel von Osteoarthrose-Patienten regelhaft basisches Kalziumphosphat ablagern.

Die zentrale Frage, welche Auslöser und Mechanismen in den ruhenden, jedoch sehr stoffwechselaktiven artikulären Chondrozyten zum Umschalten auf das embryonale Programm führen und welche Unterschiede zur Embryogenese bestehen, ist bisher weitgehend unverstanden. Interessante jüngste Ergebnisse aus unserer Gruppe legen nahe, dass im Knorpel gebundene lösliche Faktoren, wie z.B. bestimmte Chemokine, aktiv zur Aufrechterhaltung der Knorpelhomöostase benötig werden. Gehen diese im Alter, durch Belastung oder Knorpeldegradation verloren, kann dies zum Verlust der phänotypischen Stabilität artikulärer Chondrozyten führen und möglicherweise auch Entzündungsprozesse im Gelenk auslösen bzw. fördern.

Diese (sekundäre) entzündliche Komponente der Osteoarthrose ist von hoher klinischer Bedeutung, da sie (als aktivierte Arthrose) die Symptomatik der Erkrankung dominieren kann und zudem einen zentralen Unterschied zu den Vorgängen während der Embryogenese darstellt. Zudem legen - allerdings kontrovers diskutierte - Daten nahe, dass rezidivierende Entzündungsschübe die Progression der Erkrankung bestimmen und sogar z.T. Subtypen der Osteoarthrose definieren können. Die Frage, ob aus diesem Grund antientzündliche Strategien, wie sie zur Behandlung primär entzündlicher Gelenkerkrankungen eingesetzt werden, auch für die Osteoarthrose von Nutzen sind, kann derzeit nicht klar beantwortet werden; die einfache Übertragung solcher Ansätze auf die Osteoarthrose hat in bisherigen Untersuchungen allerdings zu keinen eindeutig positiven Effekten geführt. Neben verbesserten Möglichkeiten, die Schmerzen bei Osteoarthrose zu behandeln, zielen aktuelle Forschungsansätze daher vor allem darauf ab, aufbauend auf einem besseren Verständnis der erwähnten pathogenetischen Mechanismen Therapeutika zu entwickeln, die direkt in den Krankheitsprozess eingreifen, indem sie die phänotypische Stabilität der Chondrozyten sichern und ihre Regenerationskapazität wiederherstellen. Die Heterogenität der Erkrankung, die Dissoziation von Schmerz und strukturellen Defekten und die Er- 
arbeitung valider Parameter für die Beurteilung des Therapieerfolgs stellen Herausforderungen auf dem Weg zu evidenzbasierten medikamentösen Therapieoptionen dar.

Interessenkonflikt: Der Autor erklärt, dass keine Interessenkonflikte bestehen.

\section{Literatur}

1 Pap T, Korb-Pap A. Cartilage damage in osteoarthritis and rheumatoid arthritis - two unequal siblings. Nat Rev Rheumatol 2015; DOI: 10.1038/nrrheum.2015.95

\section{Bibliografie}

DOI http://dx.doi.org/10.1055/s-0035-1558062

Drug Res 2015; 65, Suppl. 1: S11-S12

(c) Georg Thieme Verlag KG Stuttgart · New York .

ISSN 2194-9379

Korrespondenzadresse

Prof. Dr. Thomas Pap

Direktor

Institut für Experimentelle

Muskuloskelettale Medizin (IEMM)

Universitätsklinikum Münster

Domagkstr. 3

48155 Münster

thomas.pap@uni-muenster.de

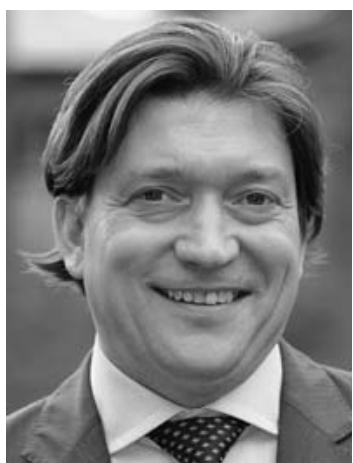

